\title{
Photometric redshifts from reconstructed QSO templates
}

\author{
Tamás Budavári ${ }^{1,2}$, István Csabai ${ }^{1,2}$, Alexander S. Szalay ${ }^{2}$, Andrew J. Connolly ${ }^{3}$, Gyula P. \\ Szokoly $^{4}$, Daniel E. Vanden Berk ${ }^{5}$, Gordon T. Richards ${ }^{6}$, Michael A. Weinstein ${ }^{6}$, Donald P. \\ Schneider $^{6}$, Narciso Benítez ${ }^{2}$, J. Brinkmann ${ }^{7}$, Robert Brunner ${ }^{8}$, Patrick B. Hall ${ }^{9,10}$, Greg \\ Hennessy $^{11}$, Željko Ivezić ${ }^{9}$, Péter Z. Kunszt ${ }^{2}$, Jeffrey A. Munn ${ }^{11}$, R. C. Nichol ${ }^{12}$, Jeffrey R. Pier ${ }^{11}$, \\ and Donald G. York ${ }^{13}$
}

\begin{abstract}
From SDSS commissioning photometric and spectroscopic data, we investigate the utility of photometric redshift techniques to the task of estimating QSO redshifts. We consider empirical methods (e.g. nearest-neighbor searches and polynomial fitting), standard spectral template fitting and hybrid approaches (i.e. training spectral templates from spectroscopic and photometric observations of QSOs). We find that in all cases, due to the presence of strong emission-lines within the QSO spectra, the nearestneighbor and template fitting methods are superior to the polynomial fitting approach. Applying a novel reconstruction technique, we can, from the SDSS multicolor photometry, reconstruct a statistical representation of the underlying SEDs of the SDSS QSOs. Although, the reconstructed templates are based on only broadband photometry the common emission lines present within the QSO spectra can be recovered in the resulting spectral energy distributions. The technique should be useful in searching for spectral
\end{abstract}

\footnotetext{
${ }^{1}$ Department of Physics, Eötvös University, Budapest, Pf. 32, Hungary, H-1518

${ }^{2}$ Department of Physics and Astronomy, The Johns Hopkins University, 3701 San Martin Drive, Baltimore, MD 21218

${ }^{3}$ Department of Physics and Astronomy, University of Pittsburgh, Pittsburgh, PA 15260

${ }^{4}$ Astrophysikalisches Institut Potsdam, An der Sternwarte 16, D-14482 Potsdam, Germany

${ }^{5}$ Fermi National Accelerator Laboratory, P.O. Box 500, Batavia, IL 60510

${ }^{6}$ Department of Astronomy and Astrophysics, The Pennsylvania State University, University Park, PA 16802

${ }^{7}$ Apache Point Observatory, P.O. Box 59, Sunspot, NM 88349-0059

${ }^{8}$ Department of Astronomy, California Institute of Technology, Pasadena, CA 91125

${ }^{9}$ Princeton University Observatory, Princeton, NJ 08544

${ }^{10}$ Pontificia Universidad Católica de Chile, Departamento de Astronomía y Astrofísica, Facultad de Física, Casilla 306, Santiago 22, Chile

${ }^{11}$ U.S. Naval Observatory, 3450 Massachusetts Ave., NW, Washington, DC 20392-5420

${ }^{12}$ Dept. of Physics, Carnegie Mellon University, 5000 Forbes Ave., Pittsburgh, PA 15232

${ }^{13}$ University of Chicago, Astronomy \& Astrophysics Center, 5640 S. Ellis Ave., Chicago, IL 60637
} 
differences among QSOs at a given redshift, in searching for spectral evolution of QSOs, in comparing photometric redshifts for objects beyond the SDSS spectroscopic sample with those in the well calibrated photometric redshifts for objects brighter than 20th magnitude and in searching for systematic and time variable effects in the SDSS broad band photometric and spectral photometric calibrations.

Subject headings: quasars: general — galaxies: distances and redshifts — galaxies: photometry — methods: statistical

\section{Introduction}

In recent years, with new multicolor surveys coming on-line, the application of photometric redshifts to the analysis of the physical properties of galaxies has become increasingly popular, (see Weymann et al. (1999) for recent works). Photometric redshift estimation techniques rely on detecting the passage of continuum features within the spectral energy distribution (SED) of sources (e.g. for galaxies, the $4000 \AA$ break) across a series of photometric passbands. For objects with featureless spectra, the photometric redshift estimation is extremely difficult or impossible as the colors of these objects vary slowly (if at all) with redshift. Emission lines may, however, help if they are strong enough relative to the continuum flux to be detectable within the broadband photometry. As quasar emission lines carry a significant amount of flux (Francis et al. 1991; Richards et al. 2001a), we expect that they will provide sufficient leverage to help in the redshift prediction for lower redshift objects. In addition, it may be easier to estimate redshifts for high-redshift objects $(z \gtrsim 3)$ due to the blanketing of the Ly $\alpha$ forest (e.g. Madau (1995)). In the astronomical literature, there are a number of different approaches for estimating photometric redshifts. Most techniques can be categorized into two basic classes; those which use spectral energy distributions as spectral templates derived from models or observations (Koo 1985; Gwyn \& Hartwick 1996; Sawicki, Lin \& Yee 1997; Connolly et al. 1999; Fernández-Soto et al. 1999; Benítez 2000; Budavári et al. 1999, 2000; Csabai et al. 2000) and those which establish a direct empirical relation between colors and redshift (Connolly et al. 1995a; Wang, Bahcall \& Turner 1998; Brunner, Connolly \& Szalay 1999) using a training set.

In this paper and the companion paper by Richards et al. (2001b), we investigate these techniques and determine how successfully they may be applied to the question of the estimation of the redshifts of QSOs. In this study, we put more emphasis on the so-called template fitting method and quasar template reconstruction. In the companion paper, we discuss an empirical method that essentially assumes that quasar colors are alike at a given redshift. We use a set of $\sim 2600$ known QSOs with five band $\left(u^{\prime}, g^{\prime}, r^{\prime}, i^{\prime}, z^{\prime}\right.$; Fukugita et al. 1996; Lupton et al. 2001) Sloan Digital Sky Survey (SDSS; York et al. 2000) photometry and redshifts in the $0<z<5$ range. In the Richards et al. (2001b) paper, we study the effects caused by reddened quasars and extended objects concerning redshift outliers, and also present more examples of science that will benefit from QSO 
photometric redshifts. Observational and target selection details are also given there.

In Section 2, this study considers empirical methods to estimate redshift. In Section 3, we apply the standard template fitting technique using composite spectra, and in Section 4 and 5, we extend our analysis to include SEDs reconstructed from photometric information.

\section{Empirical approach}

The simplest way of getting photometric redshifts is empirical fitting. We assume the redshift, $z$, to be a simple function of the magnitudes or colors. Connolly et al. (1995a) used low order polynomial functions for galaxies. The fitting formula (redshift vs. colors) is derived by using a training set of photometric data and spectroscopic redshifts. After the calibration, this simple analytic function can predict redshifts for objects directly from photometric observations. While this approach has proven successful for studies of the photometric redshifts of galaxies, the spectral energy distributions of quasars differ from galaxies in many ways. In the optical and ultraviolet, a quasar spectrum consists of an approximately power law continuum together with strong emission and absorption lines. Thus quasar colors can change drastically over the space of a small interval in redshift (as an emission line passes from one passband to the next) or can remain constant with redshift due to the power-law dependency of the continuum. Given this situation, we would not expect polynomial fitting to yield accurate and reliable photometric redshifts for QSOs due to the fact that the polynomial functions used vary slowly with redshift. This preconception is borne out by the SDSS data. Fitting both quadratic and cubic relations between the colors of the quasars and their redshifts gave almost no correlation between prediction and spectroscopic redshifts.

A more adaptive technique than polynomial fitting is that of nearest neighbor (NN) mapping. This technique assigns the redshift of a source to that of the closest object in color space (with a known redshift). This approach is, essentially, a piecewise constant function. Although, as we stated above, the polynomial technique does not work, a combination of the polynomial technique and the NN fitting technique can also be applied where we find the nearest neighbors and locally fit the polynomial relation over objects within a given radius. This method can suppress any unwanted effect due to possible erroneous nearest neighbors, but would require more data. A similar empirical method is developed in the companion paper (Richards et al. 2001b), that implements an NN estimator where the reference points are derived from colors averaged over redshift bins.

Figure 1 shows the correlation between the photometric and spectroscopic redshifts from applying the NN estimator. Most of the predicted redshifts $(\sim 70 \%)$ match well the spectroscopic measurements. There are, however, a significant number of outliers (i.e. the distribution of the prediction error is clearly non-Gaussian). This is due to the color degeneracy, i.e. within the photometric uncertainties identical objects (in colors $u^{\prime}-g^{\prime}, g^{\prime}-r^{\prime}, r^{\prime}-i^{\prime}, i^{\prime}-z^{\prime}$ ) have significantly different spectroscopic redshifts. For this reason, for this figure and for all of the following spectroscopic

redshift $\left(z_{\text {spec }}\right)$ vs. photometric redshift $\left(z_{\text {phot }}\right)$ comparison figures we calculate two rms values: one 
for all of the objects $\left(\Delta_{\text {all }}\right)$ and one for the objects that have $\left|z_{\text {spec }}-z_{\text {phot }}\right|<0.3\left(\Delta_{0.3}\right)$. The former of these measures is used to estimate the effect of the contamination of a QSO photometric-redshift sample by catastrophic failures and the latter statistic is to measure the intrinsic accuracy of the photometric redshift relation for different analysis techniques. For the NN estimator these values are $\Delta_{\text {all }}=0.64$ and $\Delta_{0.3}=0.116$. Because of the degeneracy, the number of outliers $(\Delta z>0.3)$ is quite large, approximately $30 \%$. We also note that these results are for a sample of objects already known to be quasars. If the input sample includes objects that turn out not to be quasars, then this will dilute our results.

To determine whether the magnitude of a QSO might alleviate the degeneracy between the colors of QSOs as a function of redshift, we applied the nearest neighbor method in magnitude space. We find that this degeneracy is also present when using magnitudes. One hope for removing this effect may come from extending the photometric observations to longer or shorter wavelengths, e.g. using UV or IR data. Plausible reasons for the large number of redshift outliers are discussed in Richards et al. (2001b). We note that the behavior of the estimation error seems to change around $z \sim 3$ with the outliers almost completely disappearing. This is the redshift where the Lyman-break passes out of the $u^{\prime}$ filter, which introduces a strong continuum feature into the quasar SED, and at higher redshifts, the continuum blanketing of the Ly $\alpha$ forest has even more significant effect on the observed spectrum. This attenuation enhances the quality of the redshift estimation (as is the case for high redshift galaxies). It is also important to note that these empirical methods seriously break down outside the redshift range that the training set is in. Extrapolation is impossible, for example, to higher redshifts. The method also assumes that the set of objects used for training is representative of the objects studied. Finally, transformation to other filter systems is extremely hard, if not impossible, without using spectral templates.

\section{Template fitting}

Template fitting photometric-redshift methods have the advantage of not requiring a training set (we assume that the physics of the QSO SEDs is fully encoded within our distribution of templates). We do, however, require a complete spectral library that has spectral templates that cover all of the spectral types of sources within our sample and that extend over the restframe wavelength ranges that our photometric observations occupy (for high and low redshift sources). In its simplest form this template library could be the mean spectrum of a sample of SEDs. For galaxies this is not a realistic option since the continuum shape of different spectral types of galaxies varies drastically. For quasars, there are no well-defined classes of continuum spectral features, mostly because previous observations have indicated that quasars have SEDs with similar power law continua.

In an attempt to construct such a composite mean QSO spectrum, we consider those available within the astronomical literature. We tested the method with the LBQS composite (Francis et al. 1991) spectrum. In the meantime the SDSS composite spectrum (Vanden Berk et al. 2001) became 
available; in this study, we present results using this composite. In Figure 2 we plot photometric redshifts from template fitting against spectroscopic redshifts, where template colors are derived by convolving the SDSS filter functions with the SDSS composite spectrum as well as by convolving the filter functions with our reconstructed template (Figure 3; see later). The figure is similar to that found in Figure 1: the outliers are more stratified in the template fitting case and the dispersion in photometric vs. spectroscopic redshift is somewhat larger than in the NN case $\left(\Delta_{\text {all }}=0.89\right.$ and $\Delta_{0.3}=0.123$ ). Of course direct empirical methods give less astrophysical information — as opposed to template fitting that provides consistent spectral type and luminosity besides redshift - and are hard to apply to different sets of observations, e.g. a sample with a different filter set.

The template fitting estimator does not seem to be superior when compared to NN estimators (see above and also Richards et al. (2001b)) if we use a single composite SED. In some sense it can be considered worse; there are approximately $20 \%$ more outliers. It is likely, therefore, that the composite template assumed for the photometric redshift estimation is not representative of the full QSO sample, and that we might need to introduce additional quasar spectral types (e.g. corresponding to BALs, Fe II emission objects, etc.) to our analysis. Empirical estimators, like the NN method or the one discussed in the companion paper, do not assume that QSOs at all redshifts have similar restframe spectra as opposed to the template fitting technique using a composite SED. Thus the fact that the empirical estimators yield better redshifts than a single composite may also imply that restframe QSO spectra correlate with redshift. We explore these ideas in the following section.

\section{Template reconstruction}

Without having access to a complete quasar template library, we adopt a hybrid method developed by Budavári et al. (1999, 2000) and Csabai et al. (2000) to bring together the advantages of empirical and template fitting techniques. This method requires a training set of objects with spectroscopic redshifts and multicolor photometry to establish a statistical representation of the underlying QSO SEDs. The templates are created to yield simulated colors in the best possible agreement with the photometry, and using them for photometric redshift estimation is just one application. The method is also suitable for detecting possible differences between the spectroscopic and photometric flux calibrations and to detect whether the introduction of different QSO spectral types are needed.

We propose a novel technique, similar to what is known as learning vector quantization (Kohonen 1995) in the neural network literature, that reconstructs discrete SEDs in an iterative way. The procedure called adaptive spectrum quantization (hereafter ASQ) is so robust that it can develop a set of template spectra from scratch, i.e. from one single constant function. The idea here is to separate quasars into coherent classes of spectral templates based on their photometry and spectroscopic redshifts. The algorithm improves the templates step by step to increase the agreement with the photometric observations. Since the method is capable of adding and removing classes, it 
can be started from a single class. One loop of our iterative procedure consists of four steps:

a.) All objects in the training set are categorized into classes of the most likely templates.

b.) The estimated SEDs of the objects are repaired.

c.) The reference templates are replaced with the mean of the repaired spectra.

d.) The templates are dynamically duplicated or removed if statistically desired.

Let $\Psi=\left\{\psi_{i}(\lambda)\right\}_{i=1}^{N}$ represent the initial set of spectral templates, where $N$ is the number of templates and $i=1, \ldots, N$. Given the spectroscopic redshift and multicolor photometry of an object, the most likely template can be selected from a $\chi^{2}$ optimization. Unfortunately, in general, even the best fitting template $\psi_{k}(\lambda)$ is not perfect in the sense that it cannot reproduce the measured colors if convolved with the throughput of the filters and instrument response. So the $\psi_{k}(\lambda)$ spectrum must be "repaired" according to the observational constraints (i.e. the multicolor photometry) as described in Budavári et al. (2000). The adjusted spectrum

$$
\psi_{k}^{\prime}(\lambda)=\psi_{k}(\lambda)+\delta \psi_{k}(\lambda)
$$

will be in better agreement with the measurements, but may be affected by some irregularity of the object. So instead of replacing the original $\psi_{k}(\lambda)$ with $\psi_{k}^{\prime}(\lambda)$ or adding the repaired SED to the original $\Psi$ set, one can collect all the adjusted spectra assigned to the $k$ th template and replace $\psi_{k}(\lambda)$ with the average of these repaired template spectra. A similar reparation can be applied to all templates used in the analysis.

$$
\psi_{k}(\lambda) \rightarrow\left\langle\psi_{k}^{\prime}(\lambda)\right\rangle=\psi_{k}(\lambda)+\left\langle\delta \psi_{k}(\lambda)\right\rangle
$$

Introducing additional reference templates requires more deliberation. One can think of several conditions when a class of objects should be split into two (e.g. based on the number of assigned objects etc.) but when the observed training set is limited in size, the choice of this condition may be crucial. When the iteration converges with a fixed number of templates (relaxation), a new reference spectral template can be added to the set if deemed necessary due to a large intrinsic scatter around the mean repaired spectrum $\left\langle\psi_{k}^{\prime}(\lambda)\right\rangle$ or due to an excess of member objects within a particular spectral class. To split a class one can make a copy of the corresponding template and perturb it slightly by shifting its spectral shape towards that of the modulated spectrum of a random member within its spectral class. A more elaborate discussion of the method will be described elsewhere (Budavári et al. 2001).

We tested the above method for galaxies — where the photometric redshift estimation techniques are well established - and it provides photometric redshifts for galaxies, that are in good agreement with those estimates based on eigentemplates. Quasar redshifts can also be predicted with accuracy. Starting from a featureless constant SED, we develop an average template that is 
very similar to the empirical SDSS composite spectrum (Vanden Berk et al. 2001) as seen in Figure 3. Our reconstructed template (derived purely from the photometric data) tracks the power-law continuum of the high-resolution spectrum to a remarkable degree of accuracy. The good correspondence between the continuum shapes shows not only the strength of the reconstruction algorithm, but also that the efforts the SDSS team made for the correct spectrophotometric calibration were fruitful.

The strongest spectral lines, such as Ly $\alpha$ (at $1216 \AA$, merged with N v at $1240 \AA)$, C IV $(1548 \AA)$, Mg II $(2798 \AA)$ are clearly visible. The H $\beta(4861 \AA)$ and the [O III] $(4959 \AA, 5007 \AA)$ lines are merged into a single feature and the blurred $\mathrm{H} \alpha$ (at $6563 \AA$ ) line is also present. The sharpness of these lines is quite surprising, given that only the broadband photometric information (a typical filter width is $1500 \AA$ ) and the value of the spectroscopic redshift were used as input parameters in the reconstruction algorithm.

For a given redshift, the photometric observation gives constraints on the possible underlying SED, since we expect to get back the measured photometric values by redshifting the SED and convolving it with the filter response function. This constraint obviously depends on the photometric system, and also the redshift of the object as the restframe spectrum is sampled at different wavelengths. Adding up the filter curves, shifted to the restframe, for all objects provides a smooth curve as a function of wavelength, shown with dashed line in Figure 3, that is proportional to the amount of information available for the determination of the shape of the composite. This is similar to what is typically shown for composites derived from spectrophotometric observations, but computed from the shape of the photometric transmission curves. The more objects at different redshifts we use to constrain the spectrum, the finer details could be recovered, e.g. the smaller can be the width of the reconstructed line. An additional effect is that short wavelength lines are constrained by higher redshift objects so that when they are transformed back to the restframe their linewidths get narrower. In Figure 3 one can see that reconstructed lines at shorter wavelength are narrower than the ones at longer wavelength.

The difference between the composite and reconstructed spectrum below $\sim 1200 \AA$ is due to

the fact that the composite spectrum is not corrected for inter-galactic attenuation. Comparing the photometric redshift estimations with the composite and the reconstructed spectrum in Figures $2 \mathrm{a}$ and $2 \mathrm{~b}$, we find little difference except at high redshifts which is due to the effect of a different handling of the inter-galactic attenuation.

\section{QSO spectral types}

Now we arrive at the last step of the ASQ algorithm where we consider whether there is evidence for more than just one spectral template. The optimal number of templates is partly determined by the size of the training set. If we divide a sample into too many subsets, there will not be sufficient objects within any one subset to constrain the reconstructed SEDs. On the other 
hand, the more spectral types we allow, the closer will be our resulting template library to the SEDs of the quasars for which we wish to predict a redshift. Our experiments show that, for the current training set, a QSO template library with 4 templates is the optimal choice. Introducing further templates does not improve the agreement of synthetic and measured colors significantly ( $\chi^{2}$ decreases less than 10\%) and the photometric redshifts do not become significantly better.

Compared to Figure 2, in Figure 4, the number of outliers at $z_{\text {phot }} \approx 0$ and 3.5 is greatly reduced and the redshift estimation improves by increasing the number of templates from one to four. Using the four ASQ templates gives $\Delta_{\text {all }}=0.77$ and $\Delta_{0.3}=0.120$, which is still above the results of empirical methods. The algorithm can be started from a single constant function or from the empirical composite SED. Both starting points lead to templates with approximately the same continuum features (see Figure 5). The only difference is that the emission lines remain sharper in the latter case. The match between the two sets is even more interesting if we remember that the two template libraries were created using significantly different initial spectra. This shows that the spectral classification of quasars into these spectral types is robust.

During the photometric redshift estimation with the template fitting method each object is assigned uniquely to a spectral template derived earlier by the ASQ algorithm. To test if there is any correlation between the redshift of the objects and their spectral class, we plot the redshift distributions of the 4 ASQ classes in Figure 6. We see a relation between type and redshift, which may have several reasons. Among other possibilities, it can be related to the different mean absolute luminosity of the classes, different mixture of BAL and other quasar types, sampling issues or may be a sign of QSO spectral evolution. It is also important to note that at different redshifts the photometric passbands sample different portions of the templates, and this can be accounted for having low redshift objects in class 3 and 4, where the highest redshift quasars are. Detailed analysis of this is subject of a future work.

We created composites for each ASQ spectral class using SDSS spectrophotometric observations. In Figure 7, we compare the resulting composites to the 4 ASQ templates. The order of the templates here and also in Figure 5 corresponds to the increasing mean redshift value of quasars in the classes. Comparing the continuum shape of the ASQ SEDs (the same power law $f_{\lambda} \propto \lambda^{-1.5}$ is plotted with dashed lines to help the comparison) suggests a trend with redshift.

Aside from the apparent redshift dependence of each class, there are at least two other distinct types of quasars which seem to be at least partially segregated into the different classes: broad absorption line quasars (BALQSOs), and low-redshift, low-luminosity quasars with host galaxy contamination.

Broad absorption features are evident in the class 1 composite spectrum, and also to a lesser extent in the class 2 composite spectrum. The BALQSOs in the quasar sample have been identified, and the majority $(71 \%)$ fall into either class 1 or class 2 . The fraction of quasars that have BAL features in their spectra for each class $(1,2,3,4)$ is $11 \%, 6 \%, 1.9 \%$ and $3 \%$ respectively. The colors of BALQSOs tend to be redder than non-BAL quasars (Menou et al. 2001; Sprayberry \& Foltz 
1992; Brotherton et al. 2000; Weymann et al. 1991), which probably accounts for their unequal separation here into the different classes.

The evidence for host galaxy contamination comes from the presence of stellar absorption lines in the composite spectra. For example, the Ca II H and $\mathrm{K}$ lines (insets in Figure 7) are clearly present in the class 4 composite, and weakly present in the class 3 composite. There is no evidence for them in either the class 1 or class 2 composites. Host galaxy contamination is likely to be a significant contributor to the reddening of quasars and other AGN at wavelengths beyond about $5000 \AA$ (Vanden Berk et al. 2001). For redshifts less than 1, the quasars in classes 3 and 4 are significantly less luminous than their counterparts in classes 1 and 2, and the quasars with the lowest redshifts tend to belong to classes 3 and 4 . This points to host galaxy contamination in the spectral light of the low-redshift quasars in classes 3 and 4, and is likely the cause of their being preferentially separated into those classes.

Now we return to the problem of the photometric degeneracy (i.e. quasars with the same photometric colors can have significantly different redshifts). Signs of the photometric degeneracy of quasars is visible in the photometric redshift prediction process. The $\chi^{2}$, roughly the weighted distance of the observed and estimated flux as a function of redshift $z$ has multiple minima due to the degeneracy in photometry. Two outliers from the photometric redshift relation are shown in Figure 8. Both have significant minima at the spectroscopic redshift.

In Figure 9 we plot what one would get if one could break this degeneracy by using further observations (e.g. UV) or through the use of priors that can crudely constrain the possible redshift of an object. The photometric redshift estimation algorithm can find all the local minima of the $\chi^{2}$ curve, hence we could have more redshift candidates for an object to choose from. Given the best 3 local minima for each object, we selected the one having the closest value to the spectroscopic redshift; this somewhat idealized case is plotted in the figure.

\section{Conclusions}

The success of our attempt to determine redshifts of quasars from SDSS multicolor photometry, along with the companion Richards et al. (2001b) paper, is encouraging. We can obtain redshift estimates for $70 \%$ of the QSOs with accuracy $\Delta z_{\text {rms }} \approx 0.1$. The method discussed in this paper is not only able to obtain redshifts, but also to estimate luminosities and SEDs for QSO candidates without spectroscopy. Photometric redshifts are ideally suited for many astrophysical and astronomical studies, such as the luminosity function or gravitational lensing.

Our spectral template reconstruction appears to be very robust, the algorithm yields similar sets of spectral templates whether started from a constant function or from a composite spectrum of spectrophotometric measurements. Individual spectral lines have significant effects on quasar colors. These narrow (compared to photometric bands) features contribute to photometric observations sufficiently that they can be recovered by our reconstruction technique. It is quite reassuring that 
the spectral templates reconstructed from the very low resolution photometric data show the well known emission lines of quasars. This high resolution is possible because of the large number of SDSS objects used in the template reconstruction process. Detailed analysis of the templates can give interesting information on quasar spectral features. We found that the use of multiple templates in the redshift estimation improves the photometric redshift relation and the resulting spectral types correlate with redshift.

The significance of the spectral lines in broadband photometry also means that potential errors in the boundary of the assumed passbands may result in more serious problems in the photometric redshift estimation than is the case for galaxies where broadband spectral features dominate. If the edge of a photometric filter were off just by the width of a spectral line, then the colors, hence the estimated photometric redshifts, would change substantially. The fact that the reconstructed spectral lines match well the location of the actual features is an indication that the filter curves are indeed accurately calibrated. For surveys with both photometric and spectroscopic observations (like SDSS), our method is well suited to cross-check calibration (e.g. to photometrically calibrate spectroscopic observations) or may be used to detect systematic drifts in annual, seasonal, etc. data sets.

The template reconstruction method can be extended to derive photometrically calibrated SEDs for objects fainter than the limit where spectrophotometric observations are available. Rough photometric redshift estimates can be also used in the algorithm as long as there are no systematic deviations, only scatter around the true value. An iterative process could be developed to fine tune the templates. It could be especially useful for faint high redshift QSOs, where spectroscopy is not available, but the Ly $\alpha$ attenuation could give a fairly good redshift estimate.

The Sloan Digital Sky Survey (SDSS) is a joint project of The University of Chicago, Fermilab, the Institute for Advanced Study, the Japan Participation Group, The Johns Hopkins University, the Max-Planck-Institute for Astronomy (MPIA), the Max-Planck-Institute for Astrophysics (MPA), New Mexico State University, Princeton University, the United States Naval Observatory, and the University of Washington. Apache Point Observatory, site of the SDSS telescopes, is operated by the Astrophysical Research Consortium (ARC). Funding for the project has been provided by the Alfred P. Sloan Foundation, the SDSS member institutions, the National Aeronautics and Space Administration, the National Science Foundation, the U.S. Department of Energy, the Japanese Monbukagakusho, and the Max Planck Society. The SDSS Web site is http://www.sdss.org/. I.C. and T.B. acknowledge partial support from the MTA-NSF grant no. 124 and the Hungarian National Scientific Research Foundation (OTKA) grant no. T030836. A.S. acknowledges support from NSF (AST9802980) and a NASA LTSA (NAG53503). A.J.C. acknowledges partial support from NSF grants AST0096060 and AST9984924 and an NASA LTSA grant NAG5 8546. 


\section{REFERENCES}

Benítez, N., 2000, ApJ, 536, 571

Brunner, R.J., Connolly, A.J., \& Szalay, A.S., 1999, ApJ, 516, 563

Budavári, T., Szalay, A.S., Connolly, A.J., Csabai, I., \& Dickinson, M.E., 1999, in Photometric Redshifts and High Redshift Galaxies, eds. R.J. Weymann, L.J. Storrie-Lombardi, M. Sawicki, \& R. Brunner, (San Francisco: ASP), 19

Budavári, T., Szalay, A.S., Connolly, A.J., Csabai, I., \& Dickinson, M.E., 2000, AJ, 120, 1588

Budavári, T., et al., 2001, in preparation

Connolly, A.J., Csabai, I., Szalay, A.S., Koo, D.C., Kron, R.G., \& Munn, J.A., 1995a, AJ, 110, 2655

Connolly, A.J., Budavári, T., Szalay, A.S., Csabai, I., \& Brunner, R.J., 1999, in Photometric Redshifts and High Redshift Galaxies, eds. R.J. Weymann, L.J. Storrie-Lombardi, M. Sawicki, \& R. Brunner, (San Francisco: ASP), 13

Csabai, I., Connolly, A.J., Szalay, A.S., \& Budavári, T., 2000, AJ, 119, 69

Fernández-Soto, A., Lanzetta, K.M., \& Yahil, A., 1999, ApJ, 513, 34

Francis, P.J., Hewett, P.C., Foltz, C.B., Chaffee, F.H., Weymann, R.J., Morris, S.L., 1991, ApJ, 373, 465

Fukugita, M., Ichikawa, T., Gunn, J.E., Doi, M., Shimasaku, K. \& Schneider, D.P. 1996, AJ, 111, 1748

Gwyn, S.D.J., \& Hartwick, F.D.A., 1996, ApJ, 468, L77

Kinney, A.L., Calzetti, D., Bohlin, R.C., McQuade, K., Storchi-Bergman, T., \& Schmitt, H.R., 1996, ApJ, 467, 38

Kohonen, T., 1995, Self-Organizing Maps, Springer Series in Information Sciences

Koo, D.C., 1985, AJ, 90, 148

Lupton, R.H., et al., 2001, in preparation

Madau, P., 1995, ApJ, 441, 18

Richards, G.T., et al., 2001a, AJ, 121, in press

Richards, G.T., et al., 2001b, this volume

Sawicki, M.J., Lin, H., \& Yee, H.K.C, 1997, AJ, 113, 1 
York, D.G., et al., 2000, AJ, 120, 1579

Vanden Berk, D.E., et al., 2001, in preparation

Wang, Y., Bahcall, N., \& Turner, E.L., 1998, AJ, 116, 2081

Weymann, R.J., Storrie-Lombardi, L.J., Sawicki, M., \& Brunner, R., (editors), 1999, Photometric Redshifts and High-Redshift Galaxies (San Francisco: ASP) 


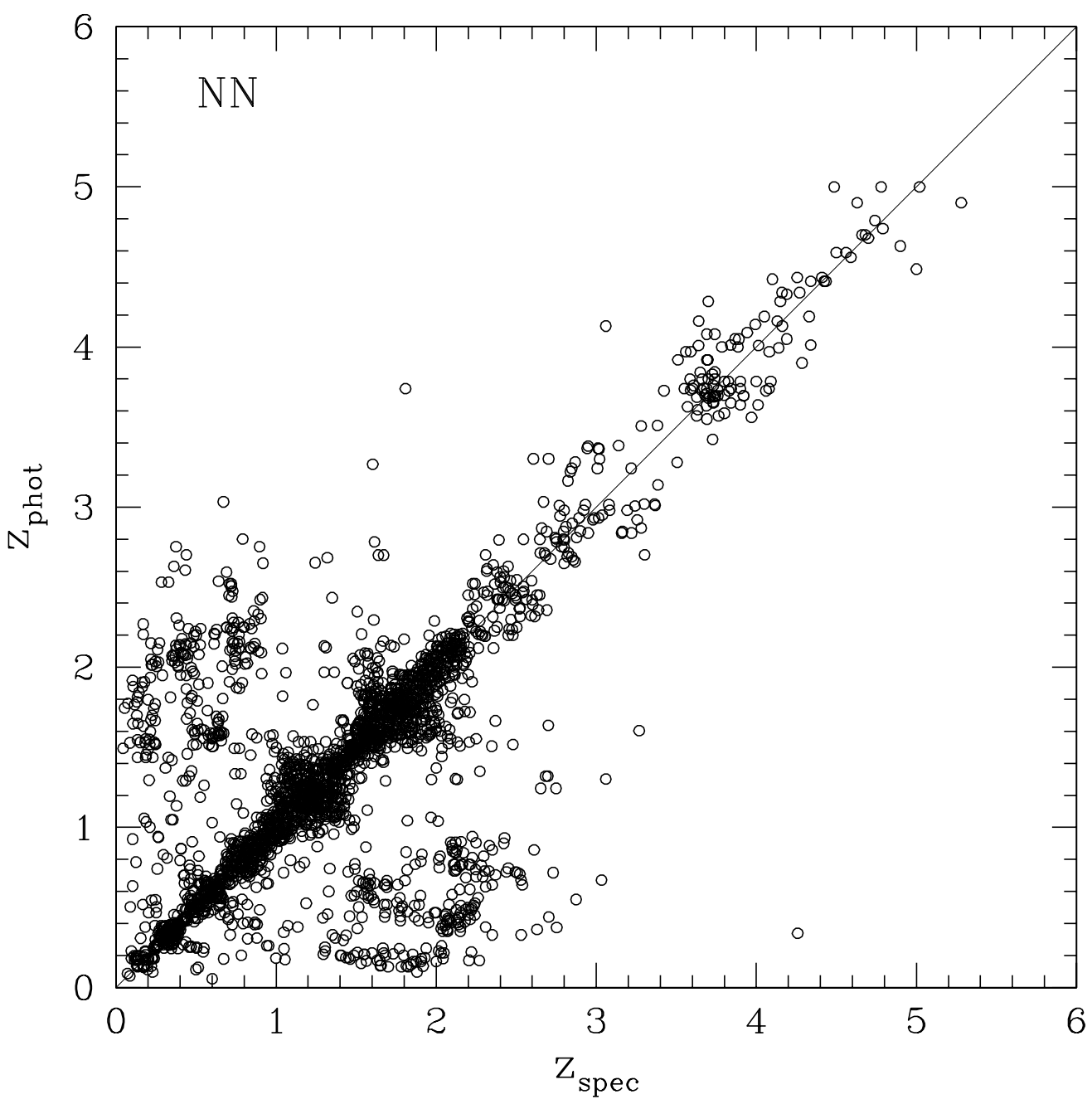

Fig. 1.- Photometric redshifts from the nearest neighbor estimator. The bulk of the objects have accurate redshifts without any systematic deviation, but the photometric degeneracy confuses quite a few estimates. The rms error is $\Delta_{\text {all }}=0.64$ for all objects and $\Delta_{0.3}=0.116$ when excluding outliers (see text). 

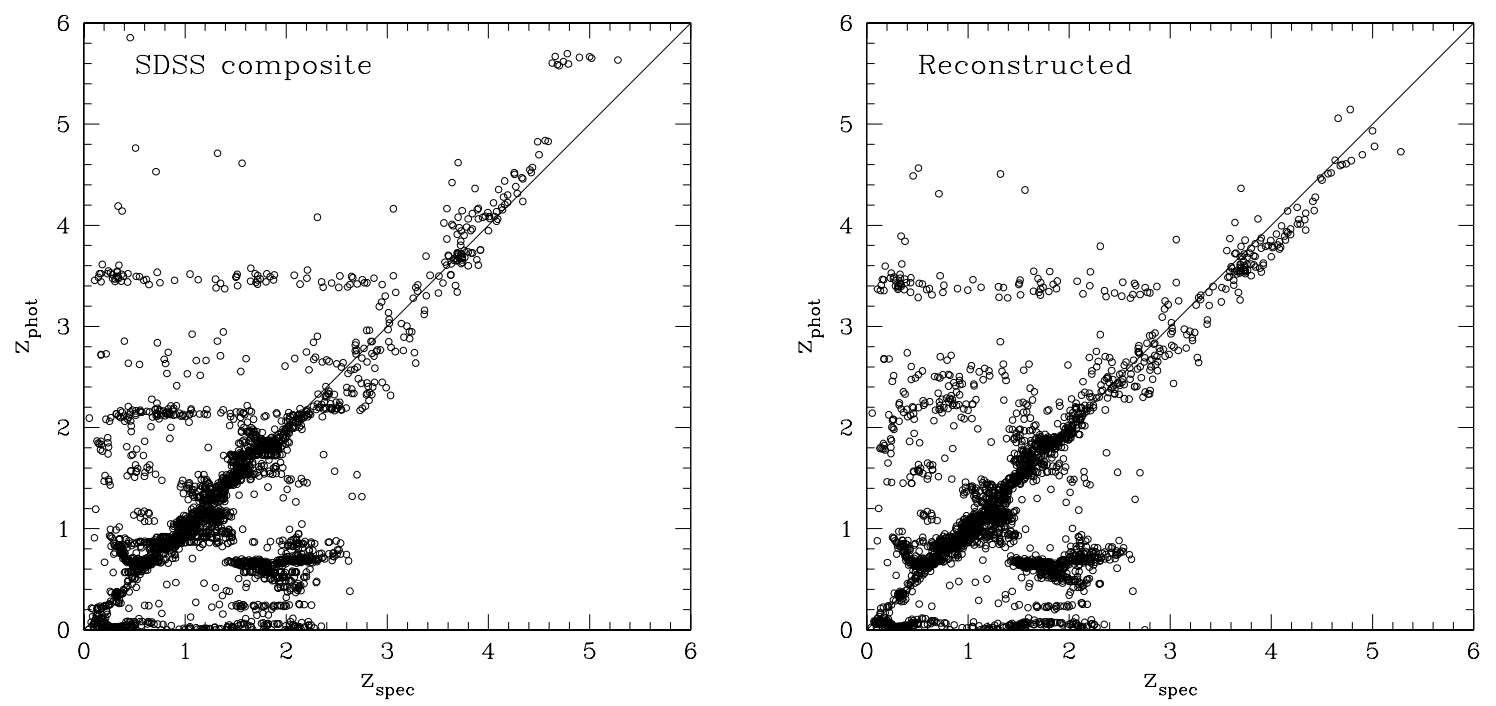

Fig. 2.- Photometric redshifts from one single SED. The SDSS composite based estimates are seen in the left (rms errors $\Delta_{\text {all }}=0.84, \Delta_{0.3}=0.128$ ) and results from the reconstructed template in the right panel $\left(\Delta_{\text {all }}=0.89, \Delta_{0.3}=0.123\right)$. 


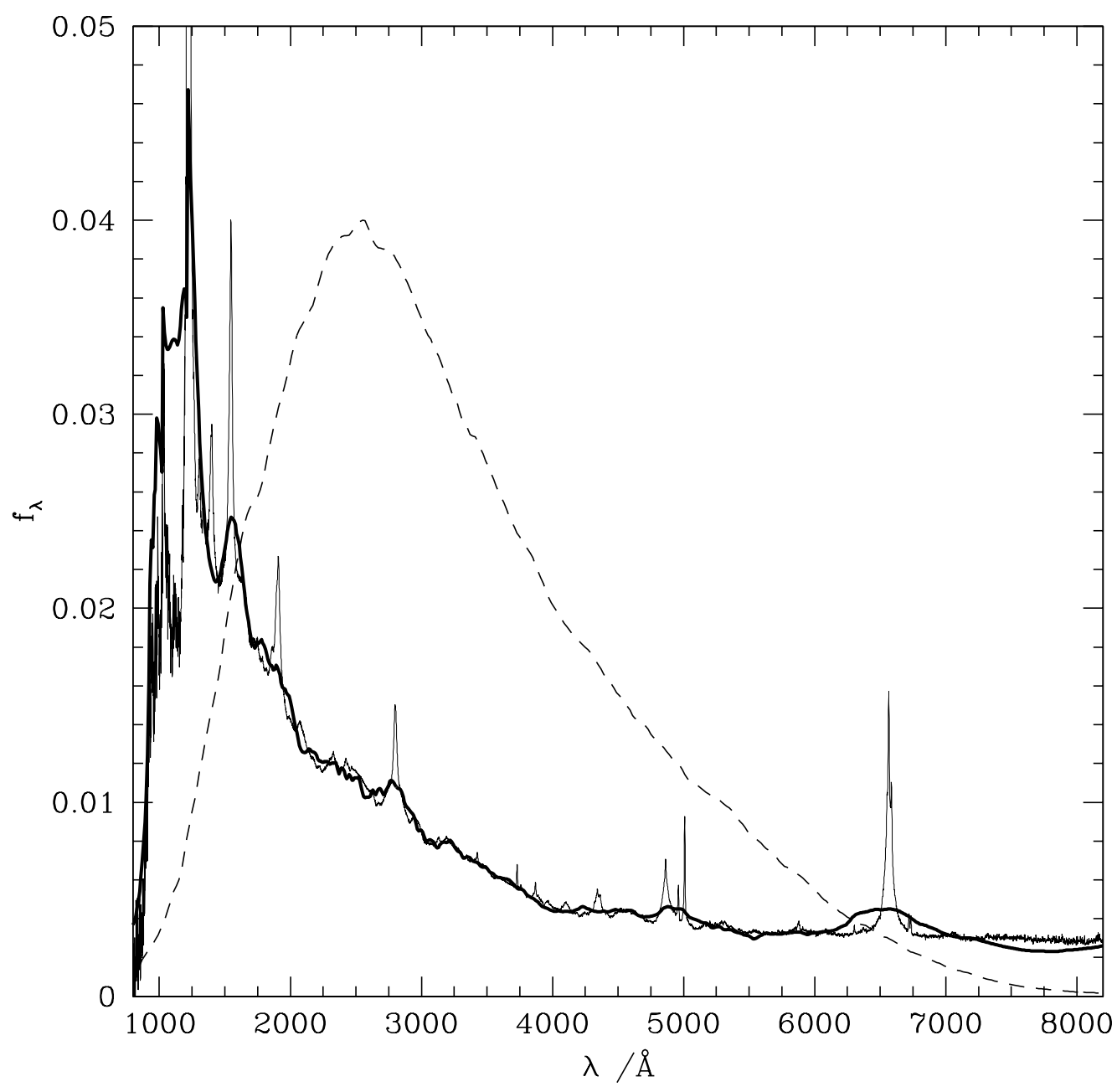

Fig. 3. - Comparison of composite SEDs. The SDSS composite QSO spectrum (thin line) and an average SED derived from photometry and redshifts of SDSS quasar sample are seen (thick line). The power-law continuum shape of the trained SED tracks nicely the thin line and also the most powerful spectral features (e.g. Ly $\alpha, \mathrm{C}$ IV, Mg II) are seen. The dashed line is the scaled constraint curve (see text for details). 


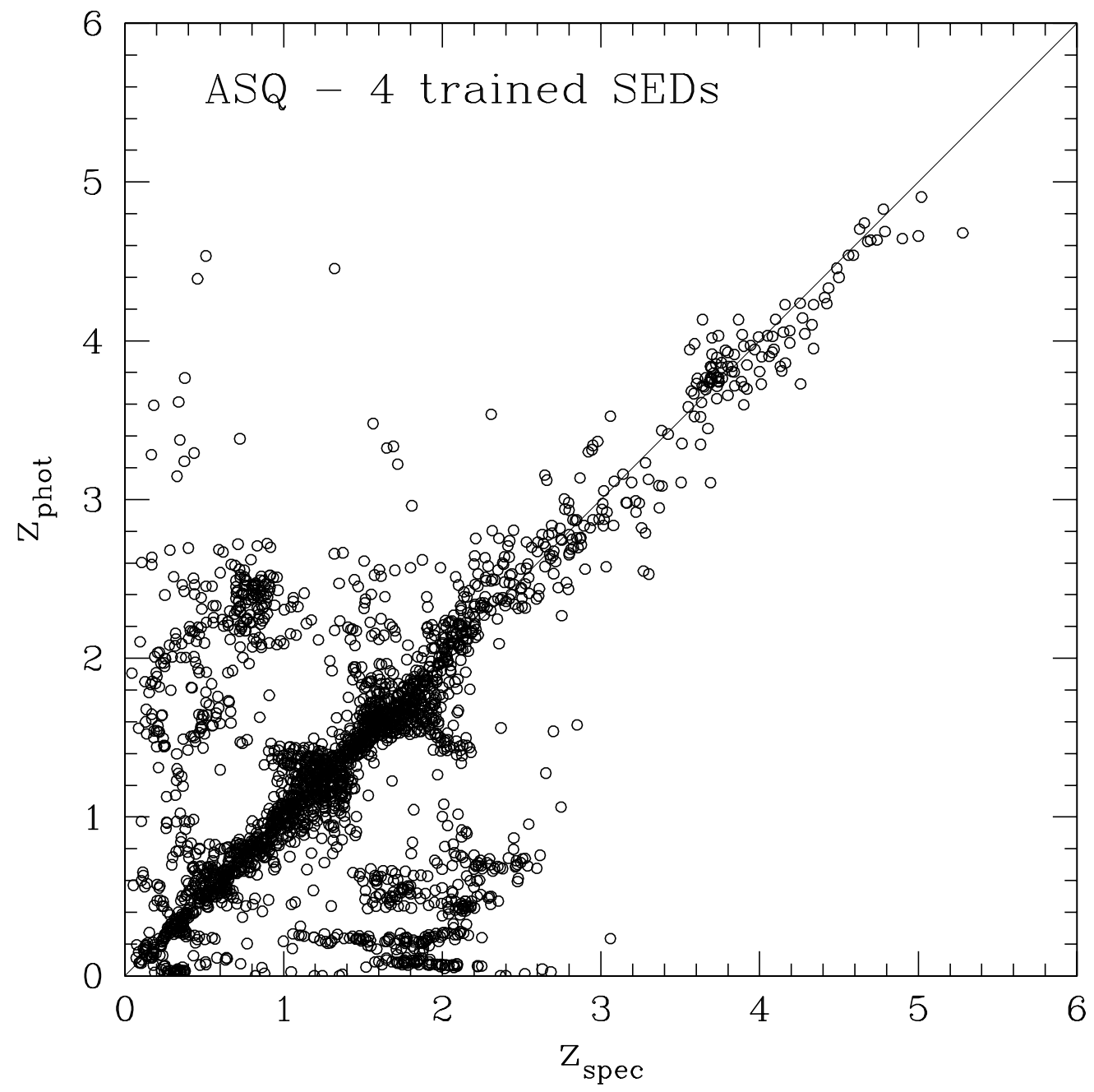

Fig. 4. - The ASQ training yields templates, that provide reliable redshift estimates. Here we plot the results using four reconstructed templates $\left(\Delta_{\text {all }}=0.77, \Delta_{0.3}=0.120\right)$. The degeneracy is clearly present in the plot just like in the NN estimates. 

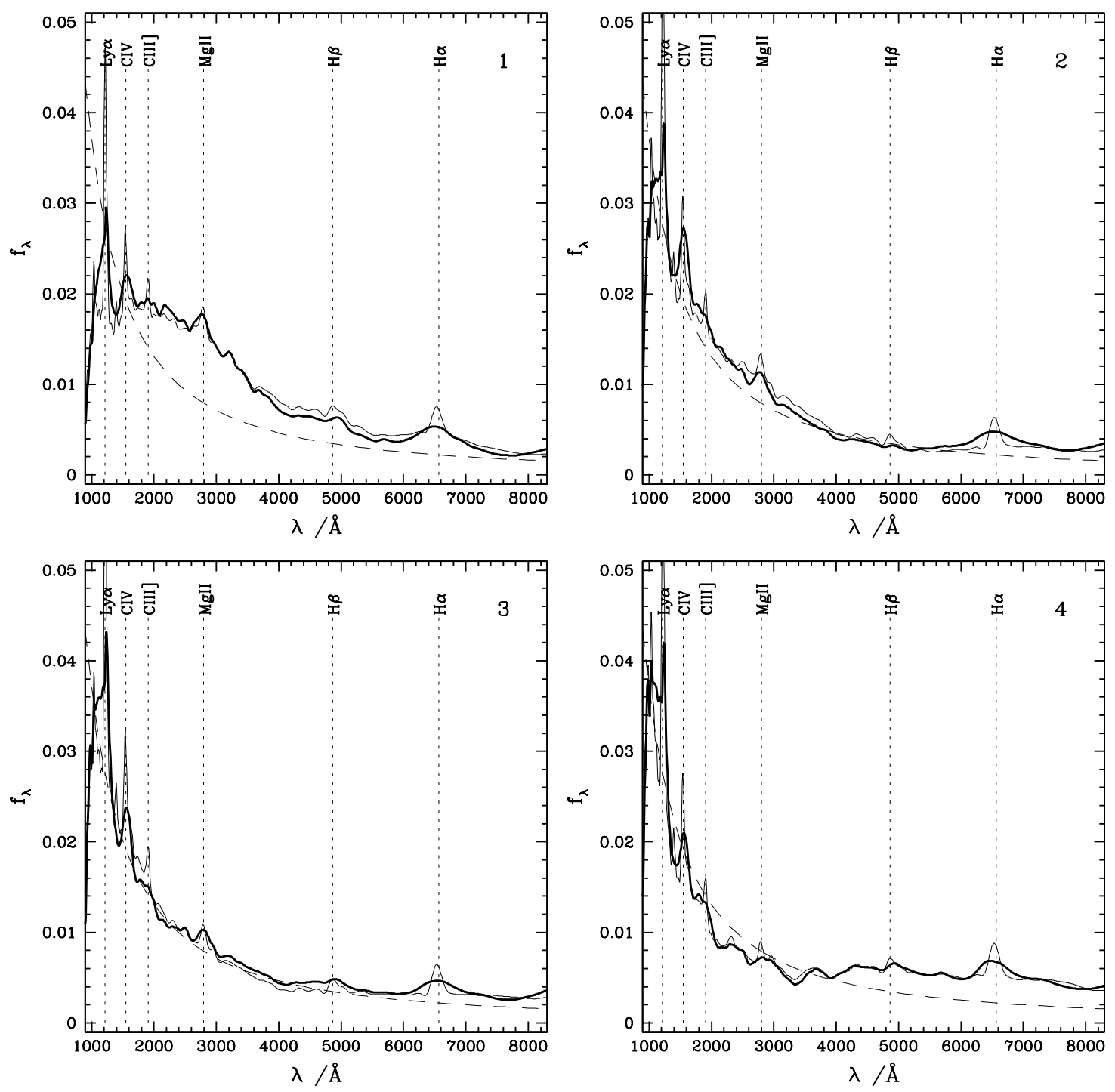

Fig. 5.- Four templates reconstructed from a constant initial SED (thick line) and from the composite SDSS SED (thin line). Despite the different initialization, the reconstruction algorithm converges to similar sets of templates. The strongest known quasar lines (markers) appear in the reconstructed spectra. The same power law curve $\left(f_{\lambda} \propto \lambda^{-1.5}\right)$ is plotted in all panels with dashed line to guide the eye to see the differences. 


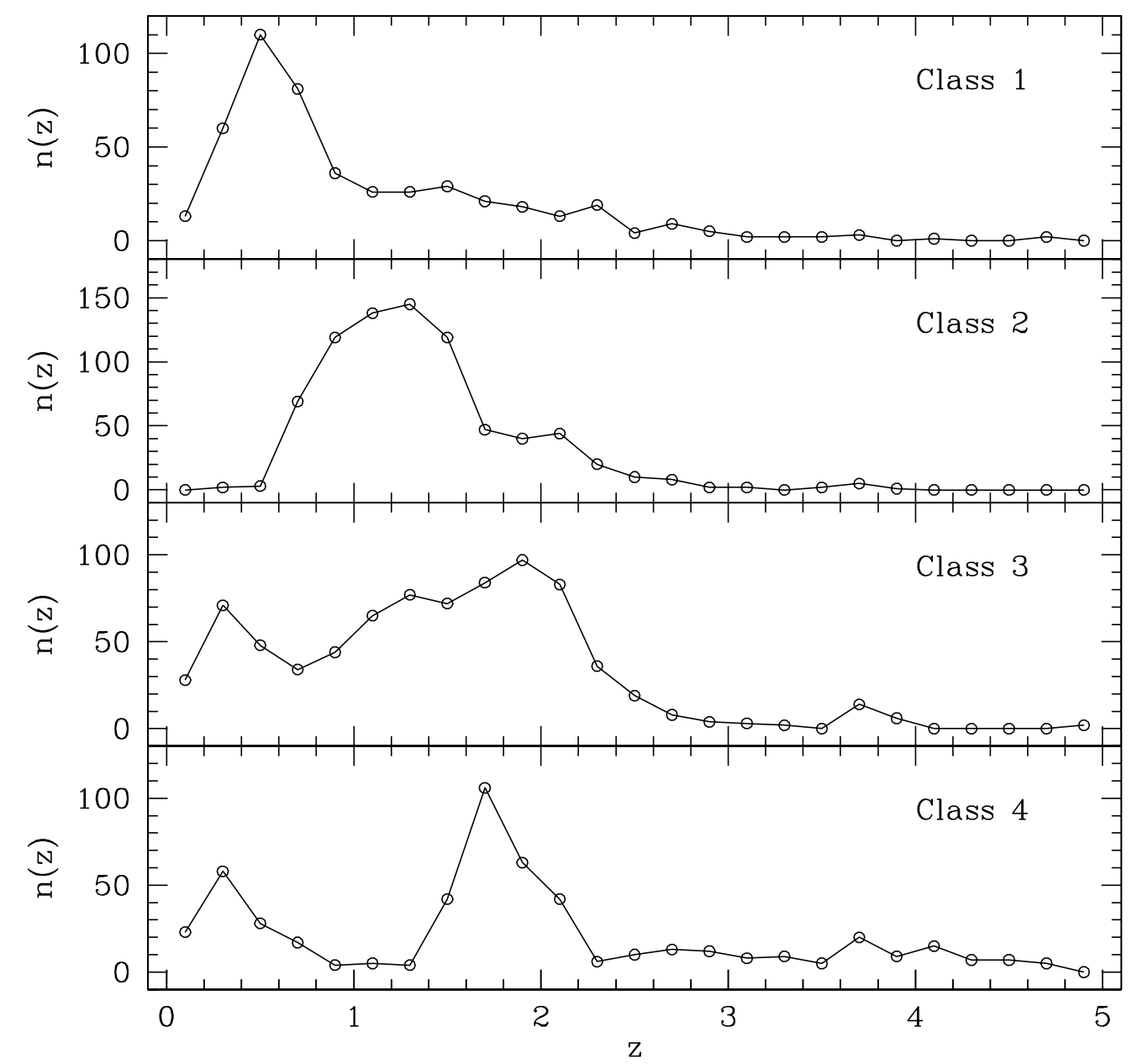

Fig. 6.- Redshift distribution of objects in the four ASQ template classes. 

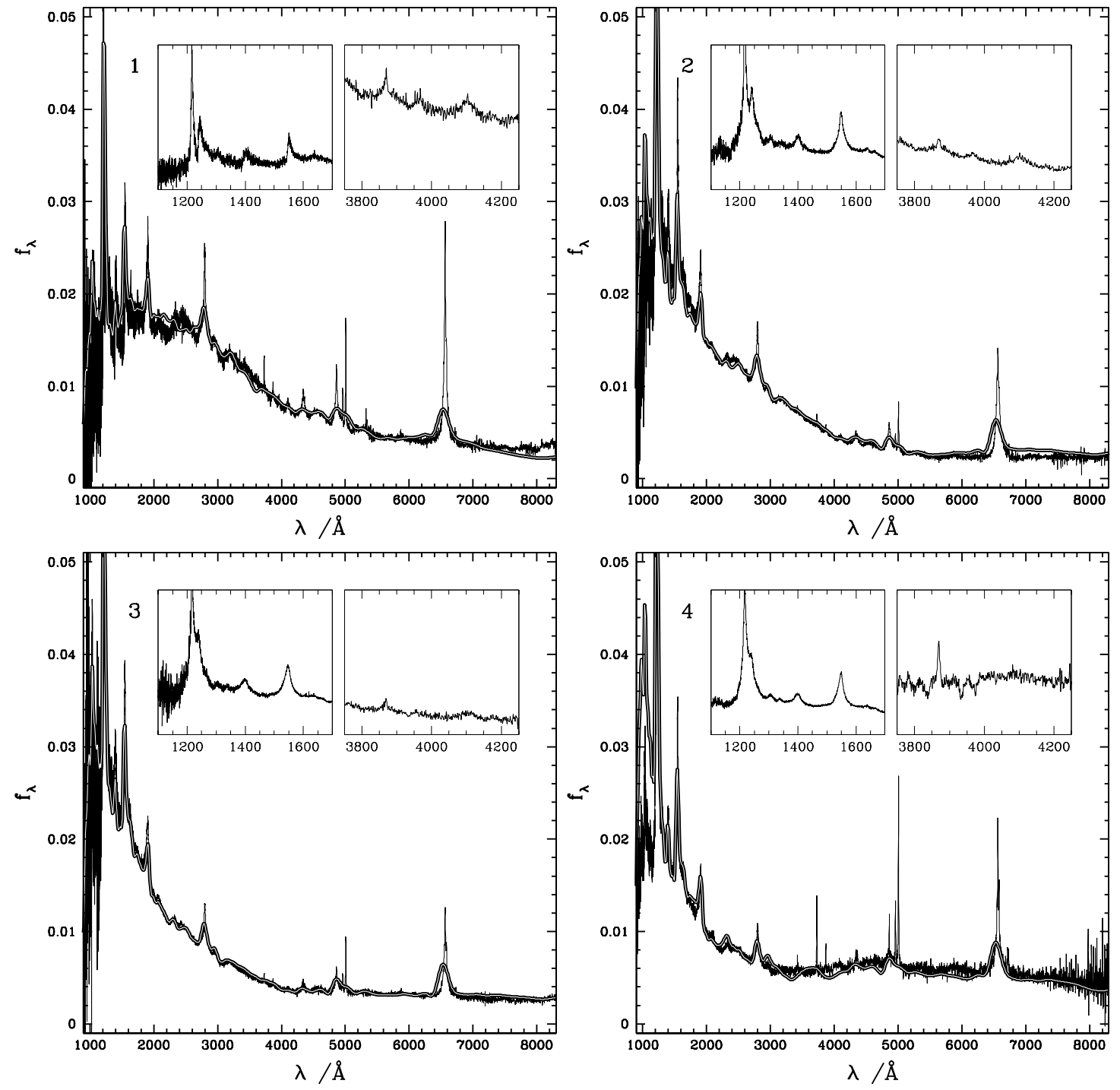

Fig. 7.- In this figure, we compare the ASQ templates (white lines) to composites created for the corresponding classes using SDSS spectra (thin lines). See discussion in text. The insets zoom on the Ly $\alpha$ to $\mathrm{C}$ IV region and the Ca II $\mathrm{H}$ and $\mathrm{K}$ lines. 

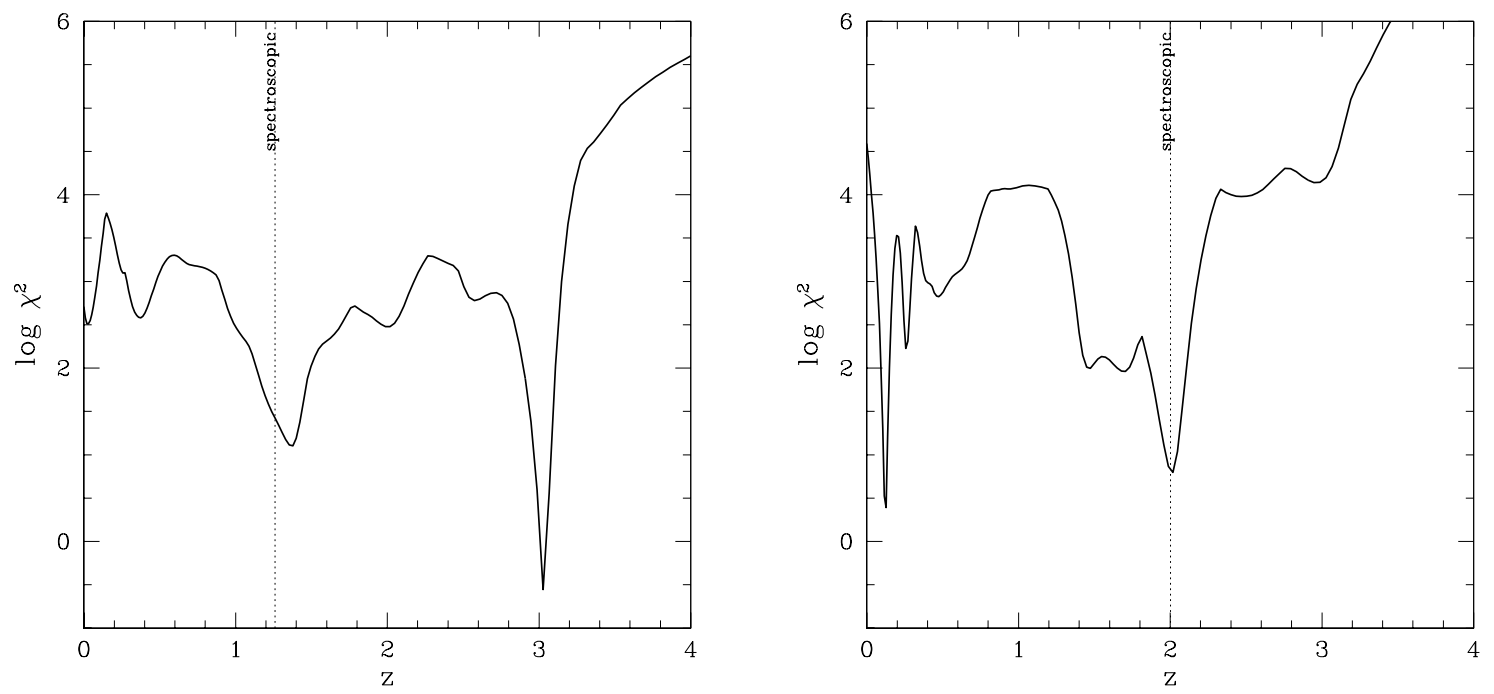

Fig. 8.- The $\chi^{2}$ curve describes the agreement of the most likely template and the photometric measurements at an assumed redshift. The figure illustrates the degeneracy in photometry by plotting $\chi^{2}$ vs. $z$, where more minima are present. For outliers the algorithm gives a false redshift value at the global minimum, but there is also a significant local minimum at the true spectroscopic redshift (vertical dotted lines). 


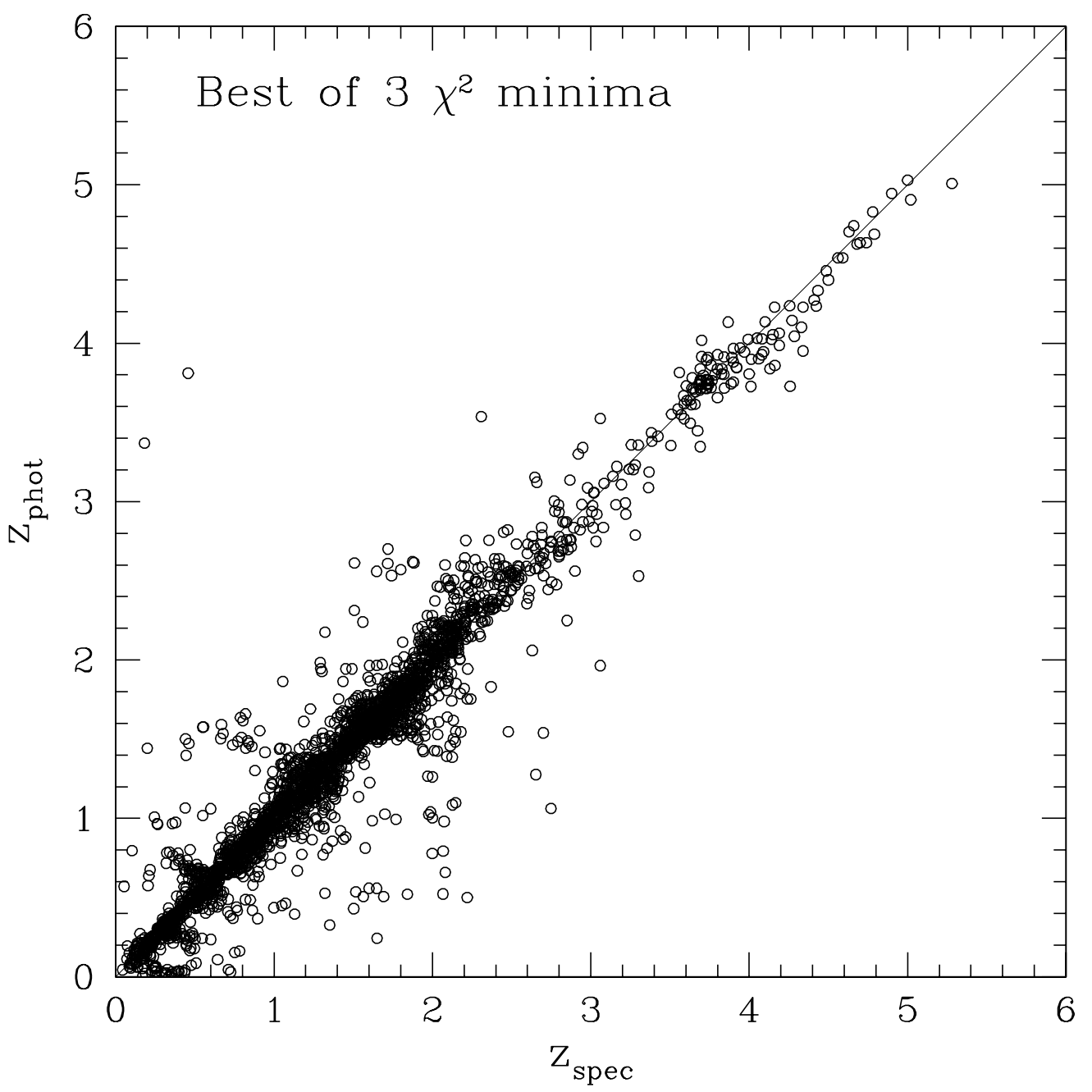

Fig. 9.- The same ASQ templates (see Figure 4) could provide much better redshift estimates, if the photometric degeneracy could be broken e.g. by applying some prior. The figure shows a somewhat idealized case: we use the closest $z_{\text {phot }}$ value to $z_{\text {spec }}$ from the three smallest $\chi^{2}$ minima $\left(\Delta_{\text {all }}=0.238\right.$ and $\left.\Delta_{0.3}=0.104\right)$. 